\title{
SURVEYS FOR CARBON STARS IN EXTERNAL GALAXIES
}

\author{
MARC AZZOPARDI \\ Observatoire de Marseille, F-13248 Marseille, France \\ and \\ Canada-France-Hawaii Telescope, Kamuela, HI 96743, U.S.A.
}

\begin{abstract}
Prominent molecular absorption bands in the spectra of carbon stars make their detection possible, even in relatively distant external galaxies. Although extensive surveys for carbon stars have been carried out mainly in the Magellanic Clouds and the dwarf spheroidals in the Galactic halo, more distant galaxies in the Local Group and beyond have been successfully searched for this kind of object.
\end{abstract}

\section{Introduction}

AGB star populations are of special interest for the study of the morphology, stellar evolution, and kinematics of galaxies. For instance, it has been shown that the ratio of carbon stars ( $\mathrm{C}$ stars) to late-type $\mathrm{M}$ stars is very sensitive to metallicity and age. In addition, the AGB star populations are of special interest for the study of the stellar evolution, structure and kinematics of nearby systems. Consequently, a number of extensive surveys for $\mathrm{C}$ stars have been carried out in the Magellanic Clouds and the dwarf spheroidals in the Galactic halo as well as in selected regions of other nearby galaxies beyond those systems. The presence of prominent molecular bands of $\mathrm{C}_{2}, \mathrm{CN}$ and $\mathrm{TiO}$ make AGB star detection possible as far away as NGC 2403 in the M81/NGC 2403 group of galaxies.

\section{Late-Type Star Surveys}

Spectroscopic surveys for late-type stars have been successfully carried out in the Magellanic Clouds and the Galactic halo dwarf spheroidal galaxies. Photographic plates obtained either with Schmidt telescopes equipped with low-dispersion objective prisms or at the prime focus of 4-m class telescopes equipped with wide-field correctors and very low dispersion transmission 
gratings (grism or grens) have been employed in extensive surveys of these systems. Occasionally, suitable intermediate to broad bandpass filters have been used to restrict the instrumental spectral range and keep the number of overlaps as low as possible. At present, the same observing technique using CCD cameras behind focal reducers at the Cassegrain focus of 2-m to 4-m class telescopes is discovering fainter $\mathrm{C}$ stars in some of the Galactic halo dwarf spheroidals (see section 4) that escaped detection in previous photographic surveys.

Slitless transmission grating detections cannot reach AGB stars effectively beyond the more distant Galactic halo dwarf spheroidal galaxies. Hence, the only possible way to probe these objects in other nearby galaxies is the imaging technique. Based on the Palmer \& Wing (1982) survey technique, intermediate-band photometric color systems measuring $\mathrm{CN}$ and $\mathrm{TiO}$ molecular bands have been defined (Cook \& Aaronson 1989; Richer et al. 1985) to identify more distant $C$ and late-M stars. However, subsequent spectroscopic observations are mandatory to confirm the nature of the AGB-star candidates detected in this manner.

Carbon stars can be identified mainly through their near-infrared CN bands at 7945,8125 and $8320 \AA$. However, they have also been successfully found by means of their Swan $\mathrm{C}_{2}$ bands at 4737,5165 and $5636 \AA$ in the blue-green spectral domain. The two survey techniques are basically complementary (see McCarthy 1987). Note that the most luminous C stars, with strong stellar winds responsible for high mass-loss rates, can only be detected at infrared or radio wavelengths.

Further information on $\mathrm{C}$ star surveys in external galaxies is provided by Azzopardi (1994) in the proceedings of the third CTIO/ESO workshop.

\section{Magellanic Clouds Surveys}

Early spectroscopic surveys for field C stars were carried out in the Large Magellanic Cloud (LMC) by Sanduleak \& Philip (1977) using, in the bluegreen spectral domain, the thin prism attached to the Curtis Schmidt at Cerro Tololo Inter-American Observatory (CTIO), and by Westerlund at Mount Stromlo Observatory using, in the near infrared, the Uppsala Southern Station Schmidt telescope (Westerlund et al. 1978). These led to the detection of 474 and 302 candidates, respectively, leading to a total number of about 700 probable $\mathrm{C}$ stars found in this system. These surveys were limited to stars brighter than $V \approx 16.5$, close to the typical magnitude of the fainter carbon stars in the LMC, so that completeness was not assured. Note that Westerlund's attempt to identify C stars in the Small Magellanic Cloud (SMC) using the same instrumentation and survey technique did not succeed. 
The near-infrared photographic transmitting - grating survey for red giant stars in selected areas in the Magellanic Clouds (52 circular LMC fields of $0.12 \mathrm{deg}^{2}$ each, and 28 circular SMC fields of $0.12 \mathrm{deg}^{2}$ each plus 9 square fields of $0.38 \mathrm{deg}^{2}$ each) carried out at the prime focus of the CTIO 4-m telescope by Blanco et al. (1980) and Blanco \& McCarthy (1983, 1990) resulted in the identification of $1045 \mathrm{C}$ star candidates in the LMC and 860 such candidates in the SMC. From the integration of the C star surface densities of the selected sample fields, Blanco \& McCarthy (1983) inferred the overall surface distribution of these objects in the two Clouds and estimated at 11,000 and 2,900 the total numbers of $\mathrm{C}$ stars in the LMC and SMC, respectively.

A photographic blue-green grism survey for field $\mathrm{C}$ stars in the SMC (13 partially overlapping circular fields of $0.78 \mathrm{deg}^{2}$ each) was carried out by our team at the prime focus of the European Southern Observatory (ESO) 3.6-m telescope (Westerlund et al. 1986; Azzopardi 1993). This search led to the detection of 1707 probable $\mathrm{C}$ stars in the central regions of this galaxy, of which fewer than $12 \%$ were already known (Rebeirot et al. 1993). Using a color-magnitude diagram obtained from the photometry of the slitless grism spectra, Rebeirot and associates identified a sample of about 80 faint and relatively blue $\mathrm{C}-$ star candidates. Subsequent medium-resolution slit spectroscopy and $J H K$ photometry for 50 of them resulted in the discovery of 38 low-luminosity $\mathrm{C}$ stars with $M_{\text {bol }}$ ranging from -1.4 to -3.0 mag (Westerlund et al. 1992, 1995: see also Fig. 2 in Azzopardi 1994). The faintest objects of this sample are among the least luminous $\mathrm{C}$ stars ever found in an external galaxy and are in many ways similar to those found in the Galactic bulge (Westerlund et al. 1991).

More recent AGB star surveys are moving toward the detection of Cand $\mathrm{M}$-type stars in the outer regions of the Magellanic Clouds and the intercloud region. These intermediate-age and cool giant stars, which are representative of the age of the majority of the stellar population of the Clouds and can be found in their outer halos, are suitable for the study of the interaction dynamics of the LMC-SMC bulk system. The two-color $\left(R, B_{\mathrm{J}}\right)$ UK Schmidt Telescope (UKST) survey in the outer halos and intercloud region of the Magellanic Clouds by Demers et al. (1993) led to the identification of 57 very red stars $(14<R<17 ; B-V>1.75)$. Subsequent spectroscopy by the author and $J H K$ infrared photometry by Feast \& Whitelock (1994) resulted in the discovery of 30 proven C stars. Since then, more very red stars have been found in an extended survey of the outer regions of the Clouds using the same imaging detection technique (Kunkel et al. 1997; Demers et al. 2000), and the C-star nature of more than 500 objects has been confirmed, the spectra being of sufficient quality to provide accurate radial velocities $\left( \pm 5 \mathrm{~km} \mathrm{~s}^{-1}\right)$. 
The blue-green medium-dispersion objective-prism UKST survey of the outer parts of the SMC by Morgan \& Hatzidimitriou (1995) resulted in the detection of $1185 \mathrm{C}$ star candidates. From this sample these authors came to the conclusion that the total number of $\mathrm{C}$ stars in the SMC would be about 3060, in good agreement with the estimates 2900 and 3100 by Blanco \& McCarthy (1983) and Azzopardi \& Rebeirot (1991), respectively. The surface distribution of the C stars found by Rebeirot et al. (1993), together with the new ones found by Morgan \& Hatzidimitriou, shows a low-eccentricity elliptical pattern with major axis parallel to the Bar, and this supports the assertion by Hardy et al. (1989) that SMC C stars, as well as planetary nebulae, belong to a spheroidal-like system.

\section{Galactic Halo Dwarf Spheroidal Surveys}

Following the pioneering work of Demers \& Kunkel (1979), who discovered 66 very red giants in the Fornax dwarf spheroidal galaxy and suspected that some of them could be carbon stars, early photographic surveys for this kind of object were carried out in seven Galactic halo dwarf spheroidal galaxies in a manner similar to the ones carried out in the Magellanic Clouds. Carbon stars were searched for in:

- Fornax and Sculptor with the CTIO 4-m reflector by Blanco \& McCarthy (Frogel et al. 1982), as well as by Richer \& Westerlund (1983) and Westerlund et al. (1987) with the ESO 3.6-m telescope, all using transmission gratings in the near infrared;

- Draco, Leo I, Leo II and Ursa Minor, through their near-infrared CN bands, by Aaronson et al. (1982, 1983);

- Carina, thanks to a color-magnitude diagram derived from a pair of plates taken with the 3.9-m Anglo-Australian Telescope (AAT) by Cannon et al. (1981) and two objective-prism plates obtained, in the blue-green spectral domain, with the UKST (Mould et al. 1982).

Those seven dwarf spheroidals were revisited by us (Azzopardi et al. 1985, 1986), using our slitless spectroscopy technique based on the photographic detection of the blue-green $\mathrm{C}_{2}$ band, at the Canada-France-Hawaii (CFHT) and ESO (Carina, only) 3.6-m telescopes. We discovered new $\mathrm{C}$ star candidates, whose nature has been confirmed by subsequent medium-resolution slit spectroscopy. They had escaped previous surveys in all systems except Ursa Minor. The more outstanding results were obtained for Leo I (19 C stars found, only one previously known) and Fornax (29 new C stars found, where 45 were previously known). We (Azzopardi, Breysacher, Muratorio \& Westerlund) are now searching for fainter $\mathrm{C}$ stars in the Galactic halo dwarf spheroidals with the same detection technique using focal reducers and CCD cameras: 
TABLE 1. Census of field carbon stars in Galactic halo galaxies

\begin{tabular}{|c|c|c|c|}
\hline $\begin{array}{r}\text { (1) } \\
\text { System }\end{array}$ & $\begin{array}{l}(2) \\
\text { No. C Stars } \\
n(C)\end{array}$ & $\begin{array}{c}(3) \\
\text { Total Number } \\
\text { N(C) }\end{array}$ & $\begin{array}{l}(4) \\
\text { References }\end{array}$ \\
\hline LMC & 849 & 11000 & $(2)(8)(14)$ \\
\hline SMC & 1718 & 3060 & $(8)(12)(18)(19)$ \\
\hline Fornax & 104 & $\sim 115$ & $(1)(5)(13)(16)(21)$ \\
\hline Leo I & 16 & & $(7)(10)(11)$ \\
\hline Carina & 9 & 9 & $(3)(6)(10)(11)(21)$ \\
\hline Leo II & 8 & 8 & $(7)(10)(21)$ \\
\hline Sculptor & 8 & & $(5)(9)(10)(11)$ \\
\hline Sagittarius & 4 & & (20) \\
\hline Draco & 3 & & $(4)(11)$ \\
\hline Ursa Minor & 1 & & (7)(11) \\
\hline Sextans & 0 & & (15) \\
\hline Galactic bulge & 34 & & (17) \\
\hline
\end{tabular}

Column 1: Name of the system.

Column 2: Number of confirmed C stars now identified in the system.

Column 3: Estimated total number of $\mathrm{C}$ stars in the system.

Column 4: References in chronological order as follows:
(1) Aaronson \& Mould (1980)
(2) Blanco, McCàrthy \& Blanco (1980)
(3) Cannon, Niss \& Norgaard-Nielsen (1981)
(4) Aaronson, Liebert \& Stocke (1982)
(5) Frogel, Blanco, McCarthy \& Cohen (1982)
(6) Mould, Cannon, Aaronson \& Frogel (1982)
(7) Aaronson, Olszewski \& Hodge (1983)
(8) Blanco \& McCarthy (1983)
(9) Richer \& Westerlund (1983)
(10) Azzopardi, Lequeux \& Westerlund (1985)
(11) Azzopardi, Lequeux \& Westerlund (1986)
(12) Westerlund, Azzopardi \& Breysacher (1986)
(13) Westerlund, Edvardsson \& Lundgren (1987)
(14) Blanco \& McCarthy (1990)
(15) Irwin, Bunclark, Bridgeland \& McMahon (1990)
(16) Lundgren (1990)
(17) Azzopardi, Lequeux, Rebeirot \& Westerlund (1991)
(18) Rebeirot, Azzopardi \& Westerlund (1993)
(19) Morgan \& Hatzidimitriou (1995)
(20) Ibata, Gilmore \& Irwin (1995)
(21) Azzopardi (present paper) 
TABLE 2. Carbon star surveys in M 31

\begin{tabular}{lrrrrl}
\hline \multirow{2}{*}{ System } & \multicolumn{2}{c}{ - Field - } & C/late M & C/M & References \\
& $\mathrm{n}$ & arcmin & & & \\
\hline M 31 & 1 & $1.8 \times 1.1$ & $5 \mathrm{C} / 41 \mathrm{M}^{+}$ & 0.12 & Richer \& Crabtree, 1985 \\
& & & & & \\
& 2 & $2.5 \times 1.5$ & $\mathrm{C} / \mathrm{M} 5^{+}$ & 0.08 & Cook et al. 1986 \\
& & & & & \\
& 1 & $3.5 \times 2.1$ & $6 \mathrm{C} / 39 \mathrm{M} 5^{+}$ & 0.15 & Richer et al. 1990 \\
& & & & & \\
& 5 & $7.0 \times 7.0$ & $55 \mathrm{C} / 2884 \mathrm{M}$ & 0.019 & Brewer et al. 1995 \\
& & $26 \mathrm{C} / 740 \mathrm{M}$ & 0.035 & \\
& & $75 \mathrm{C} / 828 \mathrm{M}$ & 0.091 & \\
& & $82 \mathrm{C} / 727 \mathrm{M}$ & 0.113 & \\
& & $5 \mathrm{C} / 56 \mathrm{M}$ & 0.089 & \\
\hline
\end{tabular}

TABLE 3. Carbon star surveys in other nearby galaxies

\begin{tabular}{|c|c|c|c|c|c|}
\hline System & $\mathrm{n}$ & $\begin{array}{c}\text { Field - - } \\
\text { arcmin }\end{array}$ & C/late M & $\mathrm{C} / \mathrm{M}$ & References \\
\hline M 33 & 1 & $2.5 \times 1.5$ & $\mathrm{C} / \mathrm{M}^{+}$ & 1.0 & Cook et al. 1986 \\
\hline NGC 6822 & 2 & $2.5 \times 1.5$ & $\mathrm{C} / \mathrm{M}^{+}$ & 33 & Cook et al. 1986 \\
\hline NGC 205 & 1 & $1.8 \times 1.1$ & $7 \mathrm{C} / 17 \mathrm{M} 2^{+}$ & 0.41 & Richer et al. 1984 \\
\hline IC 1613 & 2 & $2.5 \times 1.5$ & $\mathrm{C} / \mathrm{M}^{+}$ & $>14$ & Cook et al. 1986 \\
\hline WLM & 1 & $2.5 \times 1.5$ & $\mathrm{C} / \mathrm{M}^{+}$ & $>14$ & Cook et al. 1986 \\
\hline NGC 300 & $i$ & $5.0 \times 3.0$ & $16 \mathrm{C} / 23 \mathrm{M} 5^{+}$ & 0.41 & Richer et al. 1985 \\
\hline NGC 55 & 1 & $5.0 \times 3.0$ & $14 \mathrm{C} / 7 \mathrm{M}^{+}$ & 2.0 & Pritchet et al. 1987 \\
\hline NGC 2403 & 1 & $1.8 \times 1.1$ & $\begin{array}{c}\text { some C stars/ } \\
\text { several M stars }\end{array}$ & $? ? ?$ & Hudon et al. 1989 \\
\hline
\end{tabular}


- Carina was observed at the ESO 3.5-m NTT with EMMI, but no further C stars were found;

- Leo II was observed at the ESO 3.6-m reflector with EFOSC and at the CFHT with MOs, and two new $\mathrm{C}$ stars were found and their nature confirmed afterwards;

- Fornax was observed at the ESO 3.5-m NTT with EMMI as well as at the CFHT with MOS, and several new faint $\mathrm{C}$ star candidates were found. Up to now, the C-star nature of 30 of them has been confirmed with MEFOS attached to the ESO 3.6-m telescope. Most are probably low-luminosity C stars like those we discovered in the SMC. With 104 C stars presently known, Fornax contains by far the largest number of these objects in the Galactic halo dwarf spheroidals.

In Table 1, these results are collected together with those secured for two more dwarf galaxies, namely Sextans (no C star found) and Sagittarius (4 $\mathrm{C}$ stars found), that have been detected more recently in the Galactic halo by Irwin et al. (1990) and Ibata et al. (1995), respectively.

\section{Other Nearby Galaxy Surveys}

Two teams have carried out photometric surveys for $\mathrm{C}$ and late-M stars in selected fields of external galaxies beyond the most distant dwarf spheroidals in the Galactic halo. M 31, M 33, NGC 6822, IC 1613 and the WolfLundmark-Melotte galaxy (WLM) were observed by Aaronson and collaborators (Aaronson et al. 1984; Cook et al. 1986) using the Steward Observatory 2.3-m telescope (see Cook \& Aaronson 1989 for more information on the photometric color system used). Meanwhile, using similar intermediate-band filters, Richer and associates (see for instance Richer, Crabtree \& Pritchet 1984) were observing M 31, NGC 205 and NGC 2403 with the CFHT, as well as NGC 300 and NGC 55 at the CTIO 4-m reflector. Tables 2 and 3 give the number and size of the fields surveyed, the $\mathrm{C}$ to late-M star ratio, and the reference paper for each system. Among those galaxies, M 31 forms the subject of several late-type star surveys, and the recent work by Brewer et al. $(1995,2000)$ is called to the reader's attention.

\section{References}

Aaronson, M., Da Costa, G.S., Hartigan, P., Mould, J. R., Norris, J. \& Stockman, H.S. 1984, ApJ, 277, L9

Aaronson, M., Liebert, J. \& Stocke, J. 1982, ApJ, 254, 507

Aaronson, M. \& Mould, J. 1980, ApJ, 240, 804

Aaronson, M., Olszewski, E. W. \& Hodge, P.W. 1983, ApJ, 267, 271

Azzopardi, M. 1993, The Messenger, No. 71, 29

Azzopardi, M. 1994, in $3^{\text {rd }}$ CTIO/ESO Workshop: The Local Group: Comparative and Global Properties, ed. A. Layden, R. C. Smith and J. Storm, ESO Conference and 
Workshop No. 51, p. 129

Azzopardi, M., Lequeux, J., Rebeirot, E. \& Westerlund, B. E. 1991, A\&A Supp., 88, 265

Azzopardi, M., Lequeux, J. \& Westerlund, B.E. 1985, $A \& A, 144,388$

Azzopardi, M., Lequeux, J. \& Westerlund, B. E. 1986, $A \& A, 161,232$

Azzopardi, M. \& Rebeirot, E. 1991, in IAU Symp. 148: The Magellanic Clouds, ed. R. Haynes and D. Milne (Kluwer), p. 71

Blanco, V. M. \& McCarthy, M.F. 1983, $A J, 88,1442$

Blanco, V. M. \& McCarthy, M.F. 1990, $A J, 100,674$

Blanco, V. M., McCarthy, M. F. \& Blanco, B. M. 1980, ApJ, 242, 938

Brewer, J. P., Richer, H. B. \& Crabtree, D. R. 1995, AJ, 109, 2480

Brewer, J. P., Richer, H. B. \& Crabtree, D. R. 2000, in IAU Symp. 177: The Carbon Star Phenomenon, ed. R.F. Wing (Kluwer), p. 59

Cannon, R. D., Niss, B. \& Nørgaard-Nielsen, H. U. 1981, MNRAS, 196, 1P

Cook, K. H. \& Aaronson, M. 1989, AJ, 97, 923

Cook, K. H., Aaronson, M. \& Norris, J. 1986, ApJ, 305, 634

Demers, S. \& Kunkel, W.E. 1979, PASP, 91, 761

Demers, S., Irwin, M. J. \& Kunkel, W.E. 1993, MNRAS, 260, 103

Demers, S., Kunkel, W.E. \& Irwin, M. J. 2000, in IAU Symp. 177: The Carbon Star Phenomenon, ed. R. F. Wing (Kluwer), p. 528

Feast, M. \& Whitelock, P. 1994, MNRAS, 269, 737

Frogel, J. A., Blanco, V. M., McCarthy, M.F. \& Cohen, J. G. 1982, ApJ, 252, 133

Hardy, E., Suntzeff, N. B. \& Azzopardi, M. 1989, ApJ, 344, 210

Hudon, J. D., Richer, H. B., Pritchet, C. J., Crabtree, D., Christian, C. A. \& Jones, J. $1989, A J, 98,1265$

Ibata, R. A., Gilmore, G. \& Irwin, M. J. 1995, MNRAS, 277, 781

Irwin, M. J., Bunclark, P.S., Bridgeland, M. T. \& McMahon, R. G. 1990, MNRAS, 244, $16 \mathrm{P}$

Kunkel, W.E., Irwin, M. J. \& Demers, S. 1997, A\&A Supp., 122, 463

Lundgren, K. 1990, $A \& A, 233,21$

McCarthy, M.F. 1987, in ESO Conference and Workshop No. 27: Stellar Evolution and Dynamics of the Outer Halo of the Galaxy, ed. M. Azzopardi and F. Matteucci, p. 203

Morgan, D. H. \& Hatzidimitriou, D. 1995, A\&A Supp., 113, 539

Mould, J. R., Cannon, R. D., Aaronson, M. \& Frogel, J. A. 1982, ApJ, 254, 500

Palmer, L. G. \& Wing, R. F. 1982, AJ, 87, 1739

Pritchet, C. J., Richer, H. B., Schade, D., Crabtree, D. \& Yee, H. K. C. 1987, ApJ, 323, 79

Rebeirot, E., Azzopardi, M. \& Westerlund, B. E. 1993, A\&A Supp., 97, 603

Richer, H. B. \& Crabtree, D. R. 1985, ApJ, 298, L13

Richer, H. B., Crabtree, D.R. \& Pritchet, C. J. 1984, ApJ, 287, 138

Richer, H. B., Crabtree, D. R. \& Pritchet, C. J. 1990, ApJ, 355, 448

Richer, H. B., Pritchet, C. J. \& Crabtree, D. R. 1985, ApJ, 298, 240

Richer, H. B. \& Westerlund, B. E. 1983, $A p J, 264,114$

Sanduleak, N. \& Philip, A. G. D. 1977, Publ. Warner \& Swasey Obs., v. 2, No. 5

Westerlund, B.E., Azzopardi, M. \& Breysacher, J. 1986, A\&A Supp., 65, 79

Westerlund, B. E., Azzopardi, M., Breysacher, J. \& Rebeirot, E. 1992, A\&A, 260, L4

Westerlund, B. E., Azzopardi, M., Breysacher, J. \& Rebeirot, E. 1995, $A \& A$ A, 303, 107

Westerlund, B.E., Edvardsson, B. \& Lundgren, K. 1987, $A \& A, 178,41$

Westerlund, B. E., Lequeux, J., Azzopardi, M. \& Rebeirot, E. 1991, $A \& A, 244,367$

Westerlund, B.E., Olander, N., Richer, H.B. \& Crabtree, D. R. 1978, A $6 A$ A Supp., 31, 61 